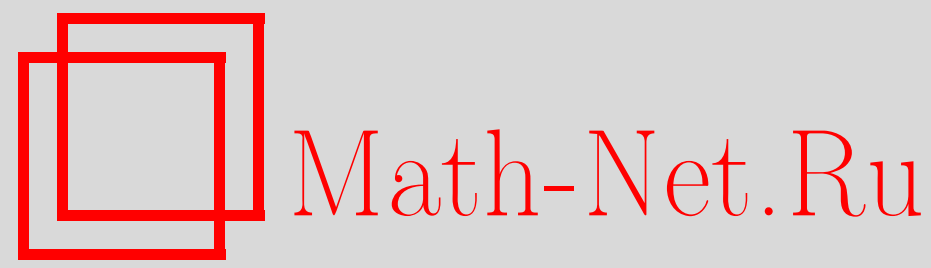

А. В. Филиновский, Интегральные оценки решений волнового уравнения в областях с бесконечной границей, Матем. заметки, 1996, том 60, выпуск 2, 310-312

DOI: https://doi.org/10.4213/mzm1831

Использование Общероссийского математического портала MathNet.Ru подразумевает, что вы прочитали и согласны с пользовательским соглашением

http://www . mathnet.ru/rus/agreement

Параметры загрузки:

IP : 44.207 .124 .84

26 апреля 2023 г., 13:55:44

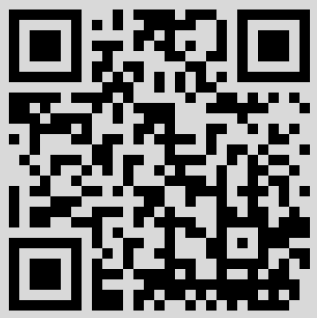




\section{ИНТЕГРАЛЬНЫЕ ОЦЕНКИ РЕШЕНИЙ ВОЛНОВОГО УРАВНЕНИЯ В ОБЛАСТЯХ С БЕСКОНЕЧНОЙ ГРАНИЦЕЙ}

\section{А. В. Филиновский}

Пусть $\Omega \subset \mathbb{R}^{n}, n \geqslant 2,-$ неограниченная область, замыкание которой не содержит начало координат, с гладкой границей $\Gamma$. Для произвольных чисел $m=1,2$, $\ldots, n-1$ и $0 \leqslant \alpha \leqslant 1$ определим функцию

$$
\varphi(x)=\alpha\left(x_{1}^{2}+\cdots+x_{m}^{2}\right)+x_{m+1}^{2}+\cdots+x_{n}^{2} .
$$

$\Omega$ называется областью класса $D_{\varphi}$, если

$$
\left(\nu_{x}, \nabla \varphi(x)\right) \leqslant 0, \quad x \in \Gamma
$$

где $\nu_{x}$ - единичный вектор внешней по отношению к $\Omega$ нормали к $\Gamma$.

$\Omega$ называется областью класса $G_{\varphi}$, если $\Omega \in D_{\varphi}$ и существует такая постоянная $C_{1}>0$, что

$$
x_{1}^{2}+\cdots+x_{m}^{2} \leqslant C_{1}|x|^{2 \alpha}, \quad x \in \Omega .
$$

При $\alpha=1$ неравенство (1) выполняется с $C_{1}=1$ для произвольной $\Omega \subset \mathbb{R}^{n}$, и класс $G_{\varphi}$ содержит все области, граница которых звездна относительно начала координат. Из (1) следует, что граница области $\Omega \in G_{\varphi}, \alpha<1$, бесконечна.

Рассмотрим смешанную задачу для волнового уравнения

$$
\begin{gathered}
u_{t t}-\Delta u=0, \quad t>0, \quad x \in \Omega \\
u(0, x)=0, \quad u_{t}(0, x)=g(x), \\
\left.u\right|_{\Gamma}=0, \quad t>0 .
\end{gathered}
$$

Начальную функцию будем предполагать вещественнозначной, гладкой в $\bar{\Omega}$ и согласованной с граничным условием (4).

Для решения задачи (2)-(4) справедлив закон сохранения энергии

$$
E_{\Omega}(t)=\int_{\Omega}\left(u_{t}^{2}+|\nabla u|^{2}\right) d x=E_{\Omega}(0), \quad t>0 .
$$

В случае $\Omega \in G_{\varphi}, \alpha=1$ энергия $E_{\Omega}(t)$ при $t \rightarrow \infty$ рассеивается на бесконечность [1], [2], т.е. для любого компакта $\Omega^{\prime} \subset \bar{\Omega}$

$$
E_{\Omega^{\prime}}(t)=\int_{\Omega^{\prime}}\left(u_{t}^{2}+|\nabla u|^{2}\right) d x \rightarrow 0, \quad t \rightarrow \infty .
$$

В областях $\Omega \in G_{\varphi}$ с $\alpha=1$ изучено и распределение энергии по $\Omega$ при больших значениях $t$. Так для $n \geqslant 3$ в [1] установлена оценка

$$
E_{\Omega_{R}}(t) \leqslant \frac{C}{(t-R)^{2}}, \quad t>R>0, \quad \Omega_{R}=\Omega \cap\{r<R\}, \quad r=|x|,
$$


а в [2] для $n \geqslant 2$ доказана сходимость интеграла

$$
\int_{0}^{\infty}(1+t)^{1-\delta} d t \int_{\Omega}\left(\left(u_{r}+u_{t}\right)^{2}+|\nabla u|^{2}-u_{r}^{2}\right) d x \leqslant C, \quad \delta>0 .
$$

В настоящей работе исследуется распределение энергии $E_{\Omega}(t)$ в областях $\Omega \in G_{\varphi}$ при $\alpha<1$.

Предположим, что

$$
\int_{\Omega} r^{2 \alpha+1}\left(g^{2}+|\nabla g|^{2}+\sum_{j, l=1}^{n}\left(\frac{\partial^{2} g}{\partial x_{j} \partial x_{l}}\right)^{2}\right) d x<\infty
$$

TEOPEMA 1. Пусть $n \geqslant 9$,

$$
\begin{aligned}
& 1 \leqslant m \leqslant 2 n+3-\sqrt{28 n+42}, \\
& \frac{1}{2}+\frac{n}{(n-2)(n-6)} \leqslant \alpha<1,
\end{aligned}
$$

$u \Omega \in G_{\varphi}$. Тогда справедлива оиенка

$$
\begin{aligned}
\int_{0}^{\infty} d t \int_{\Omega}\left(\left(\frac{(\nabla u, \nabla \varphi)}{|\nabla \varphi|}+\frac{|\nabla \varphi|}{2 \sqrt{\varphi}} u_{t}\right)^{2}+\right. & \left.|\nabla u|^{2}-\frac{(\nabla u, \nabla \varphi)^{2}}{|\nabla \varphi|^{2}}+\frac{u^{2}}{r^{2}}\right) \frac{d x}{r^{2 \alpha-1}} \\
& \leqslant C_{2} \int_{\Omega} r^{2 \alpha+1}\left(g^{2}+|\nabla g|^{2}+(\Delta g)^{2}\right) d x
\end{aligned}
$$

с постоянной $C_{2}$, зависящей от $n u \alpha$.

ДоКАЗАТЕЛЬСтво теоремы 1 основано на свойствах в замкнутой верхней полуплоскости $\{\operatorname{Im} k \geqslant 0\}$ решения $v(x, k)$ краевой задачи Дирихле для уравнения Гельмгольца

$$
\begin{gathered}
\Delta v+k^{2} v=h(x), \quad x \in \Omega, \quad k=\omega+i \mu \\
\left.v\right|_{\Gamma}=0
\end{gathered}
$$

где

$$
\int_{\Omega} r^{2 \alpha+1}|h|^{2} d x<\infty
$$

ТЕОРема 2. Пусть $n \geqslant 9, m$ и а удовлетворяют $(5),(6) u \Omega \in G_{\varphi}$. Тогда функиия $v(x, k)$ удовлетворяет неравенству

$$
\begin{gathered}
\int_{\Omega}\left(\left|\frac{(\nabla v, \nabla \varphi)}{|\nabla \varphi|}-i \omega \frac{|\nabla \varphi|}{2 \sqrt{\varphi}} v\right|^{2}+|\nabla v|^{2}-\frac{|(\nabla v, \nabla \varphi)|^{2}}{|\nabla \varphi|^{2}}+\frac{|v|^{2}}{r^{2}}\right) \frac{d x}{r^{2 \alpha-1}} \\
\leqslant C_{3}\left(\omega^{2}+1\right) \int_{\Omega} r^{2 \alpha+1}|h|^{2} d x, \quad-\infty<\omega<+\infty, \quad \mu>0
\end{gathered}
$$

c постоянной $C_{3}$, зависящей от $n, \alpha$, и равномерно непрерывна на $K_{N}=$ $\{\operatorname{Im} k>0,|k|<N\}$ в норме $W_{2}^{2}\left(\Omega^{\prime \prime}\right)$, әде $N$ - любое полохительное 
число, $а \Omega^{\prime \prime} \subset \Omega-$ произвольная ограниченная область. Для кахдого $\omega \in(-\infty,+\infty)$

$$
\lim _{k \rightarrow \omega}\|v(x, k)-v(x, \omega)\|_{W_{2}^{2}\left(\Omega^{\prime \prime}\right)}=0, \quad \operatorname{Im} k>0,
$$

где функиия $v(x, \omega)$ является решением задачи

$$
\begin{aligned}
\Delta v+\omega^{2} v & =h(x), \quad x \in \Omega, \\
v & \left.\right|_{\Gamma}=0,
\end{aligned}
$$

и удовлетворяет (7).

Из (7) следует, что при всех $\omega \in(-\infty,+\infty)$ существует решение задачи $(8),(9)$ с конечным интегралом

$$
\int_{\Omega}\left(\left|\frac{(\nabla v, \nabla \varphi)}{|\nabla \varphi|}-i \omega \frac{|\nabla \varphi|}{2 \sqrt{\varphi}} v\right|^{2}+\frac{|v|^{2}}{r^{2}}\right) \frac{d x}{r}<\infty .
$$

Теорема 3. Пусть $n \geqslant 9, m$ удовлетворяет (5), $1 / 2 \leqslant \alpha \leqslant 1 u \Omega \in D_{\varphi}$. Тогда решение задачи (8)-(10), $-\infty<\omega<+\infty$, единственно.

При $\omega \neq 0$ из (10) следует условие излучения

$$
\int_{\Omega}\left|\frac{(\nabla v, \nabla \varphi)}{|\nabla \varphi|}-i \omega \frac{|\nabla \varphi|}{2 \sqrt{\varphi}} v\right|^{2} \frac{d x}{r}<\infty .
$$

В случае $\alpha=1$ из (11) вытекает условие

$$
\int_{\Omega}\left|v_{r}-i \omega v\right|^{2} \frac{d x}{r}<\infty
$$

а при $\alpha=0, m=n-1-$ условие

$$
\int_{\Omega}\left|v_{\left|x_{n}\right|}-i \omega v\right|^{2} \frac{d x}{r}<\infty
$$

из которого для $\Omega \in G_{\varphi}, \Omega \subset\left\{x_{n} \geqslant \delta>0\right\}$ следует условие излучения Реллиха [3]

$$
\int_{\Omega}\left|v_{x_{n}}-i \omega v\right|^{2} \frac{d x}{x_{n}}<\infty .
$$

Московский государственный технический университет Поступило им. Н. Э. Баумана

\section{СПИСОК ЦИТИРОВАННОЙ ЛИТЕРАТУРЫ}

1. Zachmanoglou E. C. // Arch. Rat. Mech. Anal. 1963. V. 14. № 4. P. 312-325. 2. Муравей Л. А. // Тр. МИАН. 1988. Т. 185. С. 171-180. 3. Rellich F. // Jahresb. der Deutsch. Math. Verein. 1943. V. 53. № 1. P. 57-65. 